\title{
RAPD fingerprint construction and genetic similarity of Mesona chinensis (Lamiaceae) in China
}

\author{
G.F. Zhang, J.M. Guan, X.P. Lai, J. Lin, J.M. Liu and H.H. Xu \\ College of Chinese Medicine, Guangzhou University of Chinese Medicine, \\ Guangzhou, Guangdong, China \\ Corresponding author: X.P. Lai \\ E-mail: laixp88@126.com
}

Genet. Mol. Res. 11 (4): 3649-3657 (2012)

Received April 23, 2012

Accepted July 20, 2012

Published October 4, 2012

DOI http://dx.doi.org/10.4238/2012.October.4.12

\begin{abstract}
Mesona chinensis is an economically important agricultural crop, primarily cultivated for making grass jelly. It was originally discovered in South China. We examined 18 cultivars, including cultivars from Guangdong, Fujian, and Guangxi, China, Taiwan, and Indonesia, and a hybrid (a cross between cultivars from Indonesia and Guangdong), based on RAPD markers. The genetic similarity coefficient was calculated by NTSYS 2.10 and the clustering analysis was made by UPGMA. PCR amplification with 10 primers produced 163 bands; 94\% of the amplified loci were polymorphic. The primers S208, S206, and S253 could completely distinguish all 19 samples by constructing a DNA fingerprint. Cluster analysis divided the 19 cultivars into five groups, with an overall genetic similarity coefficient of 0.68 . Correlations were found among regional distributions, parental sources, and RAPD markers, demonstrating the rich genetic diversity of these 19 cultivars of $M$. chinensis. This study provides useful information for the classification, identification, and breeding of M. chinensis.
\end{abstract}

Key words: Mesona chinensis; RAPD fingerprint; Genetic similarity 


\section{INTRODUCTION}

Mesona chinensis, a medicinal and edible plant native to South China, India, and Malaysia, is one of the dry overground members of the genus Mesona in the family Lamiaceae (Zhao et al., 2011). It has strong adaptability and grows well with adequate water and shade. The plant has a sweet flavor and can lower body temperature, so traditional Chinese doctors use it to cure heatstroke and fever (Liu and Fang, 1998). In modern medicine, it can be used to treat diseases such as heatstroke, diabetes, hypertension, and acute nephritis (Shen et al., 2000). M. chinensis is also rich in flavones, polysaccharides, polyphenols, pigments, and amino acids, making it useful as a raw material for traditional herbal medicine, health foods, plant beverages, and daily supplements (Liu and Chen, 2004; Huang et al., 2012).

M. chinensis Benth., M. paruifsota Benth., and M. procumbens Hemsl. are known to grow in China (Wu and Wang, 2009), but the genetic resources of M. chinensis are disordered, and the pedigrees of local resources remain undefined. Identifying the genetic relationships among these species using geographic origin and morphological differences is difficult. Therefore, the use of molecular techniques in further studies of genetic identification is essential.

At present, the genetic relationships between medical plants are mainly established using DNA molecular marker techniques including random amplified polymorphic DNA (RAPD) (Arif et al., 2010), amplified fragment length polymorphism (Ghosh et al., 2011), simple sequence repeat (Xu et al., 2011), DNA barcoding (Guo et al., 2011), and so on. However, each approach has shortages and boundedness. Amplified fragment length polymorphism technology is complicated and expensive and requires DNA and incision enzymes. Simple sequence repeat techniques should be used for species for which DNA information is known; its accuracy is influenced by sequence stitching and primer design software. DNA barcode techniques are costly and so complicated that the outcome of such analyses and their displays are indirect. Comparatively, RAPD techniques have many advantages, such as low DNA requirements and the capability to use primers without genus specificity. RAPD analysis can help to construct DNA fingerprints of species for which researchers have carried out no previous molecular studies (Hammad and Qari, 2010). In brief, RAPD technique can assist in the direct analysis of the polymorphisms of medicinal plant and determination of particular DNA markers. Therefore, it can be used distinguish genetic specificity among plant populations and to develop means of identifying crude drugs from appearance to gene (Zhu et al., 2011). RAPD techniques have been used to identify many medicinal plants, such as Dendrobium officinale (Ding et al., 2009), Trollius accessions (Li and Ding, 2010), and Dalbergia sissoo (Wang et al., 2011).

Recently, changes in the M. chinensis germplasm have occurred owing to cultivar selection and natural evolution. Therefore, analyzing the diverse genetic resources of $M$. chinensis at the molecular level is necessary. Until now, we have found only one related molecular research in the literature in which the genomic DNA of M. chinensis is extracted from the 18 cultivars and one hybrid and analyzed (Guan et al., 2010). Additional investigations are still needed. The current study of various producing areas and populations of M. chinensis provides a concentrated resource through the construction of RAPD fingerprints, which offer a theoretical basis for classification, identification, and breeding. 


\section{MATERIAL AND METHODS}

\section{Plant material}

A total of 19 M. chinensis samples were collected from Guangdong (China), Fujian (China), Guangxi (China), Taiwan (China), and Indonesia. The samples included one hybrid (Table 1).

\begin{tabular}{lll}
\multicolumn{2}{c}{ Table 1. Source of materials. } & Collecting date \\
\hline Code & Source & September 14, 2009 \\
\hline N1 & Zengcheng City in Guangdong Province, China & January 2, 2010 \\
N2 & Yangchun City in Guangdong Province, China & September 24, 2009 \\
N3 & Pingyuan County in Guangdong Province, China & September 24, 2009 \\
N4 & Chaoyang District of Shantou City in Guangdong Province, China & January 2, 2010 \\
N5 & Fenghuang Mountain of Chaozhou City in Guangdong Province, China & September 26, 2009 \\
N6 & Yongan City in Fujian Province, China & December 26, 2009 \\
N7 & Yongding County in Fujian Province, China & September 26, 2009 \\
N8 & Xintian Mountain at Xinluo District of Longyan City in Fujian Province, China & January 2, 2010 \\
N9 & Xintian Village at Xinluo District of Longyan City in Fujian Province, China & September 14, 2009 \\
N10 & Loumian Village at Xiaba Country of Wuping County in Fujian Province, China & December 26, 2009 \\
N11 & Xiaba Village at Xiaba Country of Wuping County in Fujian Province, China & December 26, 2009 \\
N12 & Pingchuan Town of Wuping County in Fujian Province, China & December 26, 2009 \\
N13 & Yanqian Town of Wuping County in Fujian Province, China & December 26, 2009 \\
N14 & Shifang Town of Wuping County in Fujian Province, China & December 26, 2009 \\
N15 & Zhongbao Town of Wuping County in Fujian Province, China & October 24, 2009 \\
N16 & Lingshan County in Guangxi Province, China & December 26, 2009 \\
N17 & Guanxi Town of Xinzhu County in Taiwan Province, China & January 2, 2010 \\
N18 & Indonesia & October 24, 2009 \\
N19 & Hybrid cross Indonesia and Chaoyang District of Shantou City in Guangdong Province & \\
\hline
\end{tabular}

\section{RAPD analysis}

A modified cetyltrimethylammonium bromide method (Guan et al., 2010) was used to extract the genomic DNA from fresh leaves of $19 \mathrm{M}$. chinensis samples. The clear and repeatable bands were screened using random primers with mixed genomic DNA. Then, RAPD amplifications of $19 \mathrm{M}$. chinensis samples were performed three times.

The amplification system $(20 \mu \mathrm{L})$ was as follows: $2 \mu \mathrm{L} 10 \mathrm{X}$ buffer solution, $1.5 \mu \mathrm{L}$ $25 \mathrm{mM} \mathrm{Mg}^{2+}, 1.6 \mu \mathrm{L} 2.5 \mathrm{mM}$ deoxyribonucleotide triphosphate, $1 \mu \mathrm{L} 50 \mathrm{ng} / \mu \mathrm{L}$ DNA, $1 \mu \mathrm{L} 20$ $\mu \mathrm{M}$ primer, $0.2 \mu \mathrm{L} 5 \mathrm{U} / \mu \mathrm{L}$ Tap enzyme, and double-distilled $\mathrm{H}_{2} \mathrm{O}$. All reagents were purchased from TAKARA Company (Dalian, China), and the reaction was carried out using a DNA Engine polymerase chain reaction (PCR) instrument (BIO-RAD Co., USA). The amplification procedure was as follows: samples were denatured at $94^{\circ} \mathrm{C}$ for $5 \mathrm{~min}$, then at $94^{\circ} \mathrm{C}$ for $30 \mathrm{~s}$. They were renatured at $36^{\circ} \mathrm{C}$ for $45 \mathrm{~s}$ and then extended at $72^{\circ} \mathrm{C}$ for $1 \mathrm{~min}$. The entire procedure was repeated 40 times. The samples were then extended at $72^{\circ} \mathrm{C}$ for $10 \mathrm{~min}$ and preserved at $4^{\circ} \mathrm{C}$.

The PCR products were separated using 1.5\% agarose gel electrophoresis in $1 \mathrm{X}$ Trisacetate-ethylenediaminetetraacetic acid buffer for 30 to $60 \mathrm{~min}$ at $120 \mathrm{~V}$ and photographed with a Gel Doc-It imaging system (UVP, Bio Doc Co., USA). 


\section{Data analysis}

All gel bands in the RAPD fingerprints were scored as present (1) or absent or undistinguishable $(0)$ in each genotype for each primer. The number of the fingerprints was determined with number strings including 1 and 0 from top to bottom. The genetic similarity coefficient between materials was calculated using the NTSYS 2.10 software, and a dendrogram was constructed with the unweighted pair-group method with arithmetic average.

\section{RESULTS}

\section{Screening of RAPD primers and analysis of genotype polymorphism}

Ten primers with good polymorphism and high repeatability from 10010 -bp primers were amplified from $19 \mathrm{M}$. chinensis samples. A total of 163 bands were observed at 12-24 amplification loci on 19 electrophoretic gels, in which the lowest number of polymorphic bands was 12 from primers S207 and S252 and the most was 24 from primer S206 (Table 2). One hundred and fifty-three bands were polymorphic and the average polymorphic rate was $96.65 \%$ among 10 primers, implying rich genetic polymorphism among $19 \mathrm{M}$. chinensis samples.

Table 2. RAPD primer data and the percentage of polymorphic bands.
\begin{tabular}{llccc}
\hline Primer & Sequence (5'-3') & Amount of DNA bands & Amount of polymorphic bands & Polymorphic rate (\%) \\
\hline S202 & GGA GAG ACTC & 15 & 14 & 93.33 \\
S205 & GGG TTT GGCA & 16 & 15 & 93.75 \\
S206 & CAA GGG CAGA & 24 & 24 & 100.0 \\
S207 & GGC AGG CTGT & 13 & 12 & 92.31 \\
S208 & AAC GGC GACA & 18 & 17 & 94.44 \\
S215 & GGA TGC CACT & 14 & 13 & 92.86 \\
S238 & TGG TGG CGTT & 19 & 19 & 100.0 \\
S252 & TCA CCA GCCA & 12 & 12 & 100.0 \\
S253 & GGC TGG TTCC & 17 & 13 & 76.47 \\
S377 & CCC AGC TGTG & 15 & 14 & 93.33 \\
Total & & 163 & 153 & 93.65 \\
Average & & 16.30 & 15.30 & \\
\hline
\end{tabular}

\section{Fingerprint construction}

Figure 1 shows the amplification results from partial 10-bp random primers (S208, S206, and S253) to 19 genomic DNA samples. Seventeen polymorphic bands amplified by primer S208 distinguished the 19 samples completely. Primer S206 distinguished only 17 samples but distinguished all samples when paired with primer S253. Therefore, the DNA fingerprint of the $19 \mathrm{M}$. chinensis samples provides molecular proof for the identification of the M. chinensis germplasm. 


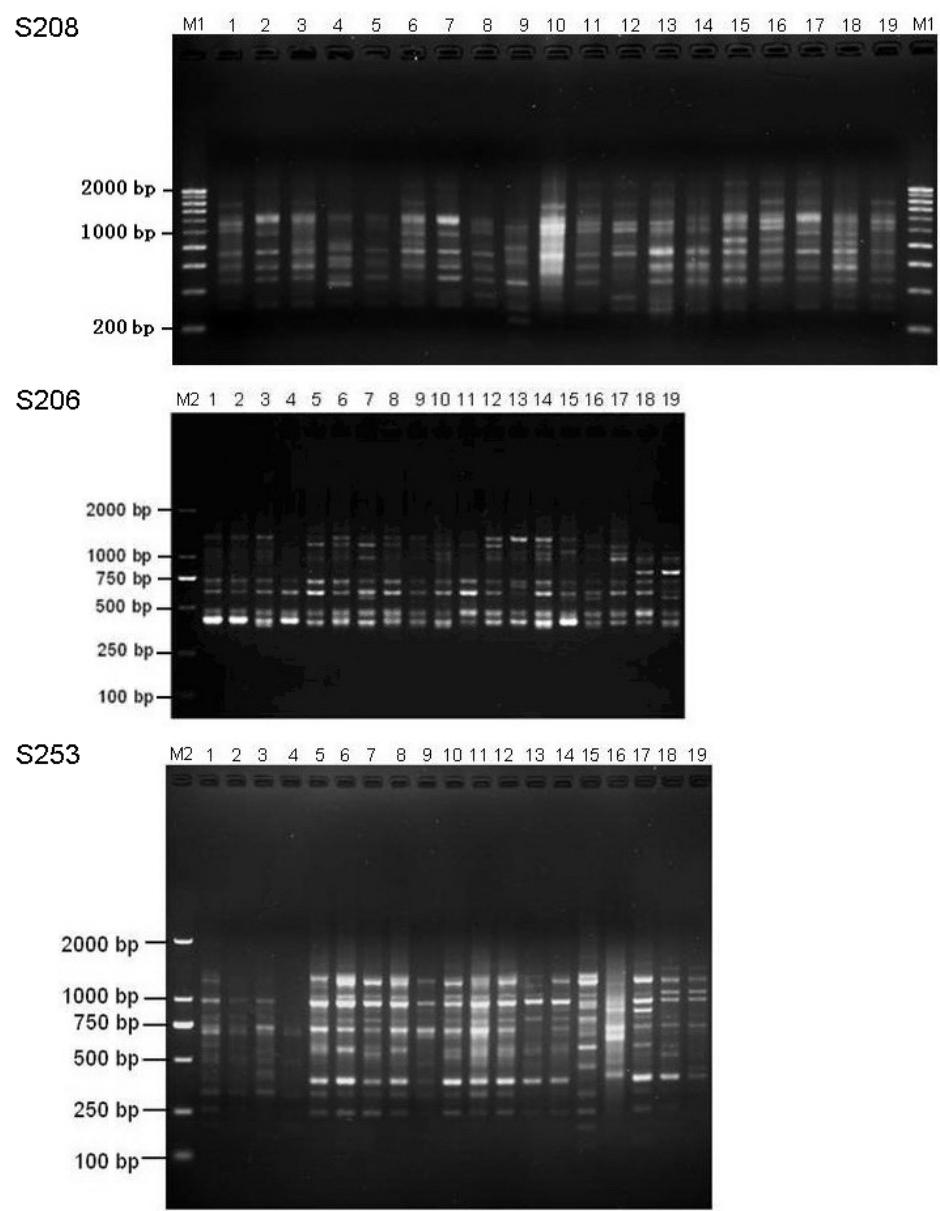

Figure 1. RAPD electrophoresis patterns of the primers S208, S206 and S253. Lane M1 = 200-bp DNA ladder; lane M2 = DL2000 DNA marker; lanes 1-19 are identified in Table 1.

\section{Analysis of genetic similarity}

According to the 163 bands amplified by the 10 primers described above, a similarity coefficient among the 19 samples was calculated using qualitative data with the similarity function in NTSYS 2.10. The value among the 19 samples ranged from 0.509 to 0.883 (Table 3 ), in which the largest value (implying the closest genetic relationship) was 0.883 between sample N12 (Pingchuan Town, Wuping County, Fujian Province, China) and sample N14 (Shifang Town, Wuping County, Fujian Province, China), whereas the lowest value (revealing the farthest genetic relationship) was 0.509, between N6 (Yongan City, Fujian Province, China) and N16 (Lingshan County, Guangxi Province, China). Table 4 shows the similarity coefficient between five regions ranging from 0.534 to 0.773 , in which the largest value was 0.773 between the Indonesia sample (N18) and the hybrid (N19), and the lowest value was 0.534 between the Guangxi Province (China) and Indonesia samples. 


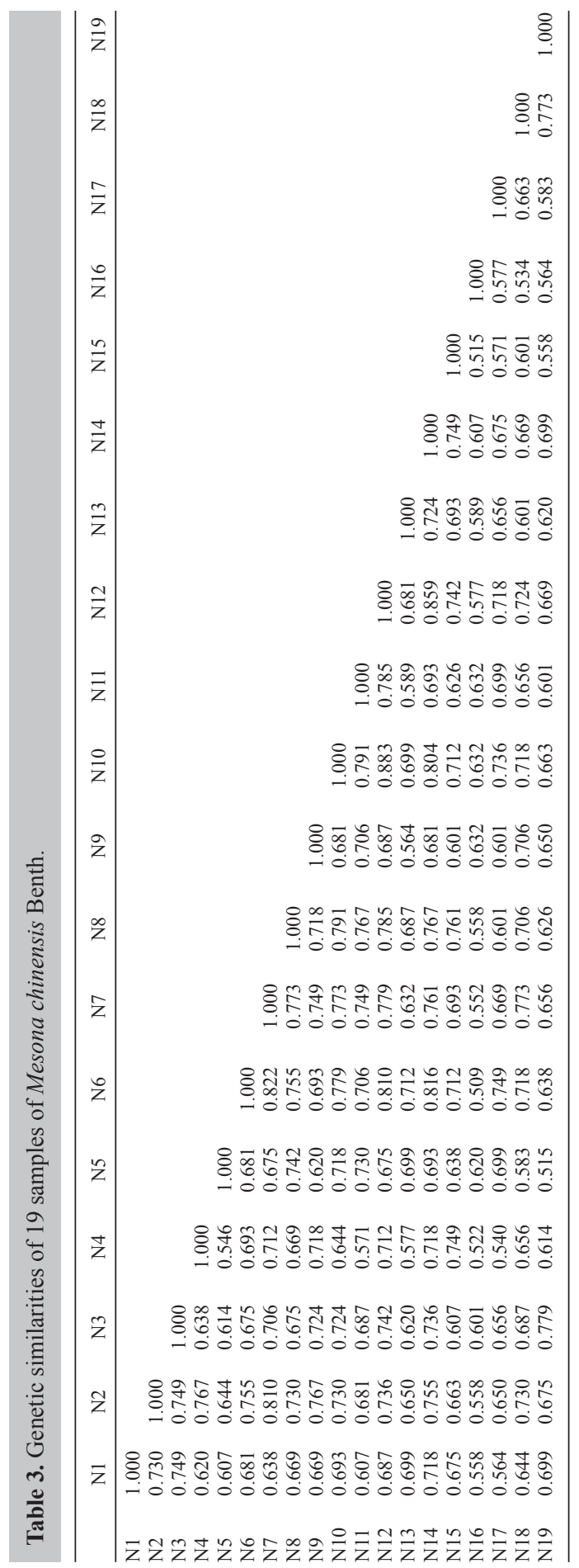


Table 4. Genetic similarities of different regions of Mesona chinensis Benth.

\begin{tabular}{lcccccc}
\hline Region & Guangdong & Fujian & Guangxi & Taiwan & Indonesia & Indonesia-Guangdong (Hybrid) \\
\hline Guangdong & 1.000 & & & & & \\
Fujian & 0.691 & 1.000 & & & & \\
Guangxi & 0.572 & 0.580 & 1.000 & & & \\
Taiwan & 0.622 & 0.668 & 0.577 & 1.000 & & 1.000 \\
Indonesia & 0.660 & 0.687 & 0.534 & 0.663 & 0.773 & 1.000 \\
Indonesia-Guangdong (Hybrid) & 0.656 & 0.638 & 0.564 & 0.583 & 0.773 \\
\hline
\end{tabular}

\section{Cluster analysis}

Figure 2 demonstrates that the 19 M. chinensis samples were distinctly clustered into five groups with a similarity coefficient cutoff of 0.68 : N1 and N13 in the first group; N3, N18, and N19 in the third group; N5 and N17 in the fourth group; N16 in the fifth group, and the rest in the second group.

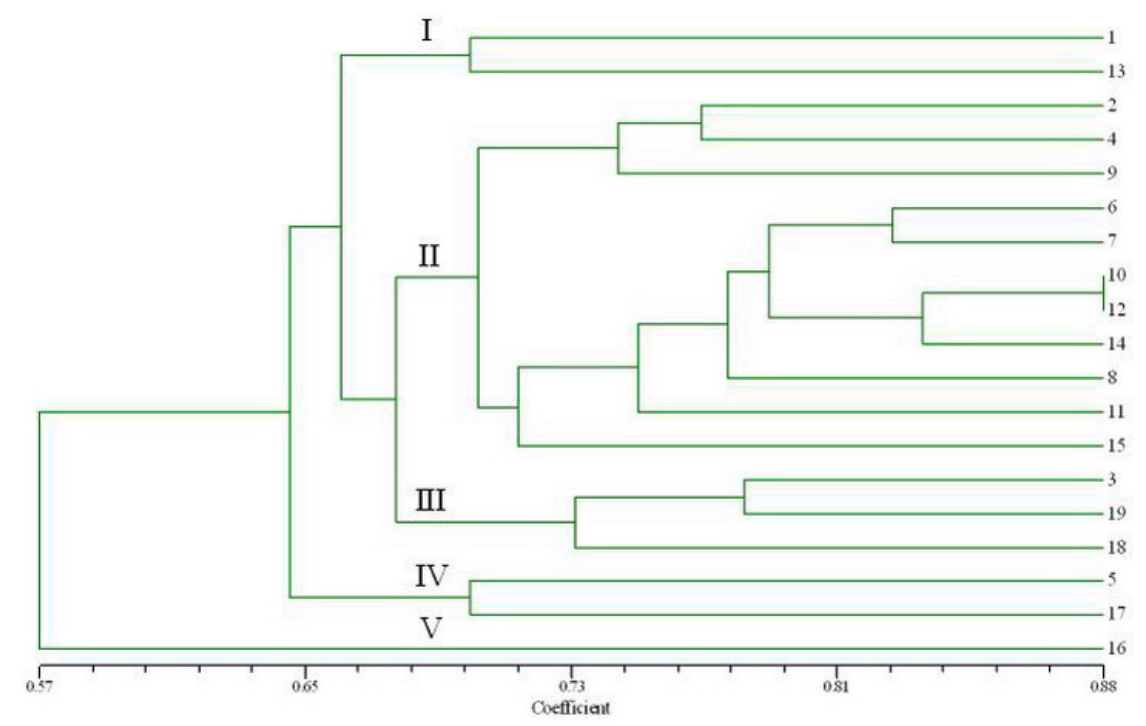

Figure 2. Dendrogram of UPGMA cluster analysis on 19 samples of Mesona chinensis Benth.

\section{DISCUSSION}

The RAPD amplification results of 19 M. chinensis samples demonstrated a high polymorphic rate, implying rich genetic diversity and strong environmental adaptability in this species, which agrees with the growth features of $M$. chinensis and its genetic trait of wide distribution (Wang et al., 2010). In the present study, primer S208 had a distinctive DNA fingerprint that could completely distinguish all of the samples, as did primer S206 combined with primer S253. Therefore, it is feasible to use an RAPD technique to construct an $M$. chinensis DNA fingerprint, which can provide molecular evidence for the identification and further exploitation of M. chinensis. 
To a certain extent, the genetic similarity coefficient reflects the degree of genetic variation and genetic basis between samples. Our RAPD investigation disclosed that the average value in $19 \mathrm{M}$. chinensis samples was 0.711, demonstrating rich genetic diversity. This diversity is not only the molecular basis of rich morphological character in M. chinensis but also the root of genetic evolution in plants.

In cluster analysis, the RAPD divisions of $M$. chinensis had a certain relationship with geographical distribution and parental source. First, the samples with close geographical distribution mostly clustered together. For example, the samples from Guangdong Province (except N1, N3, and N5) and Fujian Province (except N13) were clustered in the second group, in which both N10 and N12 from Wuping County (Fujian Province, China) first clustered together, and the sample from Guangxi Province (China) clustered solely in the fifth group, which implied a greater genetic specificity. Second, N18 and N19, which had a genetic relationship from Indonesia, clustered in the third group. However, N4 and N19 did not cluster, likely owing to the boundedness of the molecular marker technique, the results of which could not conform completely to the actual situation, and the segregation of character after hybridization, in which the genotypes of filial generation were so dispersive that they could not cluster (Zhang et al., 2009). In a word, the results of RAPD markers can provide certain reference values, such as distinguishing $M$. chinensis resources and judging genetic relationships. This molecular marker technique is an effective method for the identification of the germplasm of M. chinensis, which plays an important role in resolving the confused sources of this species.

\section{ACKNOWLEDGMENTS}

The authors wish to thank the Research Centre of Chinese Herbal Resource Science and Engineering in Guangzhou University of Chinese Medicine, China, for kindly help.

\section{REFERENCES}

Arif IA, Bakir MA, Khan HA, Al Farhan AH, et al. (2010). Application of RAPD for molecular characterization of plant species of medicinal value from an arid environment. Genet. Mol. Res. 9: 2191-2198.

Ding G, Li X, Ding X and Qian L (2009). Genetic diversity across natural populations of Dendrobium officinale, the endangered medicinal herb endemic to China, revealed by ISSR and RAPD markers. Genetika 45: 375-382.

Ghosh S, Majumder PB and Sen MS (2011). Species-specific AFLP markers for identification of Zingiber officinale, Z. montanum and Z. zerumbet (Zingiberaceae). Genet. Mol. Res. 10: 218-229.

Guan JM, Zhang GF, Lin J and Xu HH (2010). Genomic DNA extraction and analysis of Mesona chinensis Benth. J. Anhui Agric. Sci. 38: 10575-10577.

Guo X, Wang X, Su W, Zhang G, et al. (2011). DNA barcodes for discriminating the medicinal plant Scutellaria baicalensis (Lamiaceae) and its adulterants. Biol. Pharm. Bull. 34: 1198-1203.

Hammad I and Qari SH (2010). Genetic diversity among Zygophyllum (Zygophyllaceae) populations based on RAPD analysis. Genet. Mol. Res. 9: 2412-2420.

Huang GJ, Liao JC, Chiu CS, Huang SS, et al. (2012). Anti-inflammatory activities of aqueous extract of Mesona procumbens in experimental mice. J. Sci. Food Agric. 92: 1186-1193.

Li Y and Ding WL (2010). Genetic diversity assessment of Trollius accessions in China by RAPD markers. Biochem. Genet. 48: 34-43.

Liu XG and Fang YP (1998). The development and utilization of Mesona chinensis Benth. Resour. Chin. Wild Plant Res. 17: $27-30$.

Liu XG and Chen MM (2004). Research on the exploitation and utilization of Mesona Blume in China. Food Res. Dev. 25: 109-112.

Shen GL, Sun YM, Huang XY and Wu Q (2000). The research and utilization of Mesona chinensis Benth. Agric. Prod. 
Dev. 6: 8.

Wang BY, SHI L, Ruan ZY and Deng J (2011). Genetic diversity and differentiation in Dalbergia sissoo (Fabaceae) as revealed by RAPD. Genet. Mol. Res. 10: 114-120.

Wang YY, Wang CX, Huang RH, Chen WS, et al. (2010). The production technology of Mesona chinensis Benth. Guangdong Agric. Sci. 37: 86-88.

Wu LP and Wang R (2009). Progress in research and application of Mesona Blume. Str. Pharm. J. 21: 88-90.

Xu ZL, Ali Z, Yi JX, He XL, et al. (2011). Expressed sequence tag-simple sequence repeat-based molecular variance in two Salicornia (Amaranthaceae) populations. Genet. Mol. Res. 10: 1262-1276.

Zhang AS, Xu JW, Zhang LM, Xing ZF, et al. (2009). RAPD analysis of classification and genetic relationship among Northem Japonica rice. Mol. Plant Breed. 5: 885-889.

Zhao GZ, Shi YP, Huang NZ, Fu CM, et al. (2011). The research advances on Mesona chinensis Benth in China. J. South Agric. 42: 657-660.

Zhu ZH, Liu WH, Yu Q and Wan HT (2011). RAPD polymorphism of Rhizoma atractylodis macrocephalae from four natural populations. J. Zhejiang Chin. Med. Univ. 35: 575-577. 